\title{
Structures of Mn clusters
}

\author{
TINA M BRIERE*, MARCEL H F SLUITER, VIJAY KUMAR ${ }^{\dagger}$, and YOSHIYUKI KAWAZOE \\ Institute for Materials Research, Tohoku University, Aoba-ku, Sendai 980-8577, Japan \\ ${ }^{\dagger}$ Also at Dr Vijay Kumar Foundation, 45 Bazaar Street, K.K. Nagar (West), Chennai 600 078, India
}

\begin{abstract}
The geometries of several Mn clusters in the size range $\mathbf{M n}_{13}-\mathbf{M n}_{23}$ are studied via the generalized gradient approximation to density functional theory. For the 13- and 19-atom clusters, the icosahedral structures are found to be most stable, while for the $\mathbf{1 5 - a t o m}$ cluster, the $b c c$ structure is more favoured. The clusters show ferrimagnetic spin configurations.
\end{abstract}

Keywords. Clusters; magnetism.

\section{Introduction}

The beauty and challenge of modelling magnetic materials is exemplified in Mn. The dimer is weakly bound and most likely antiferromagnetic (though there is some disagreement about this), while $\mathrm{Mn}_{3}-\mathrm{Mn}_{8}$ are clearly ferromagnetic (Pederson et al 1998; Nayak et al 1998), and the most stable bulk structure, $\boldsymbol{\alpha} \mathrm{Mn}$, is an antiferromagnet. A recent Stern-Gerlach study by Knickelbein (2001) has added fresh interest to the study of $\mathrm{Mn}$. The experimental results show that clusters in the size range of 1199 atoms have significantly smaller moments per atom than ferromagnetically coupled clusters, and anomalously small moments for $\mathrm{Mn}_{13}$ and $\mathrm{Mn}_{19}$. An icosahedral growth sequence was suggested for $\mathrm{Mn}_{13}-\mathrm{Mn}_{19}$. In order to more clearly understand these results, we have studied four clusters in the size range of 13-23 atoms. The results confirm icosahedral structures for $\mathrm{Mn}_{13}$ and $\mathrm{Mn}_{19}$. Ferrimagnetic spin structures for all the clusters are preferred, in agreement with the experimental finding of relatively small net magnetic moments.

Mn clusters were studied with a planewave method employing ultrasoft pseudopotentials (Kresse and Furthmüller 1996). The generalized gradient approximation to density functional theory was used, with the exchangecorrelation potential of Perdew and Wang (1992). The cutoff energy for the plane waves was set to $283.9 \mathrm{eV}$, and reciprocal space integrations were carried out using the Gamma point. Clusters were positioned in a cubic box with an edge of $15 \AA$ (Calculations performed with a $20 \AA$ box confirmed that the energy is adequately converged at $15 \AA$ ). Structural optimizations were performed using quasi Newton-Raphson and conjugate gradient methods. The optimizations were deemed sufficiently converged when the forces were about $1 \mathrm{meV} / \mathrm{A}$. The net magnetic moments were determined by unrestricted opti-

*Author for correspondence mization after either freezing the initial net moment or specifying the initial magnetic moments on each atom. Both symmetric and asymmetric spin arrangements were considered. We defined local moments by integrating the spin density over Voronoi atomic volumes, which we refer to as 'integrated spin densities' to avoid confusion with LCAO-type local moments.

Differing geometries and spin structures were considered for each cluster size. For $\mathrm{Mn}_{13}$ and $\mathrm{Mn}_{19}$, icosahedral, decahedral, and cuboctahedral structures were examined. The hexagonal close-packed structure was also examined for $\mathrm{Mn}_{13}$. For $\mathrm{Mn}_{15}$, icosahedral, hexagonal, and $b c c$ structures were studied. For $\mathrm{Mn}_{23}$, only the icosahedron was optimized.

The most stable geometry for each cluster size is shown in figure 1. The results show that the icosahedral structure is most stable for $\mathrm{Mn}_{13}$ and $\mathrm{Mn}_{19}$, while the $b c c$ structure is preferred for $\mathrm{Mn}_{15}$. As mentioned in an earlier work (Briere et al 2002), the decahedral structures for $\mathrm{Mn}_{13}$ and $\mathrm{Mn}_{19}$ tend to deform to icosahedral, as does the cuboctahedral structure for $\mathrm{Mn}_{13} \cdot \mathrm{Mn}_{13}$ 's hexagonal close-packed structure is about $1.40 \mathrm{eV}$ higher in energy than the lowest energy icosahedron, which is in general agreement with an earlier calculation by Nayak et al (1999), though the binding energy per atom for the icosahedral structure is slightly higher $(\sim 0.07 \mathrm{eV})$ than the previous result. The most stable $\mathrm{Mn}_{15}$ icosahedral cluster is only $0.28 \mathrm{eV}$ higher in energy than the $b c c$ structure.

The binding energies for Mn clusters having 3 to 23 atoms are shown in figure 2. The energies for $\mathrm{Mn}_{3}-\mathrm{Mn}_{8}$ are taken from the work of Pederson et al (1998), who also used a gradient corrected exchange-correlation potential. Even for the largest cluster, the binding energy reaches only about $70 \%$ of the bulk. The minimum bond lengths decrease with increasing cluster size, and for $\mathrm{Mn}_{19}$ and $\mathrm{Mn}_{23}$, the shortest bond lengths are very close to those found for bulk $\alpha \mathrm{Mn}$.

Determination of the spin configurations for each cluster geometry presents a significant computational 

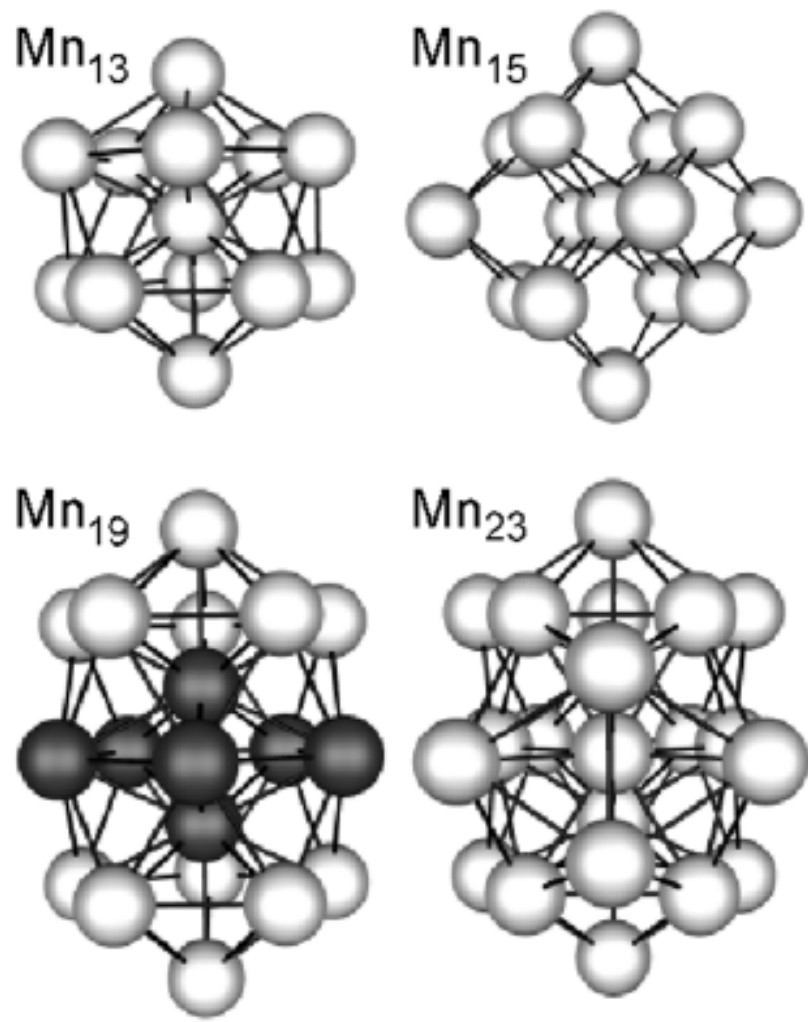

Figure 1. Geometries of the Mn clusters discussed in this work. The spin configuration of $\mathrm{Mn}_{19}$ is shown as an example. Light grey represents spin up and black, spin down.

challenge. From earlier studies (Nayak et al 1998; Pederson et al 1998), we know that small clusters have ferromagnetic coupling, and we also know there is antiferromagnetic coupling in the bulk. The small moment per atom for $\mathrm{Mn}_{11}-\mathrm{Mn}_{99}$ determined by experiment (Knickelbein 2001) indicates a transition from ferromagnetic to ferrimagnetic behaviour between $\mathrm{Mn}_{8}$ and $\mathrm{Mn}_{11}$. Thus we have considered many spin configurations, some with fixed total moments, and others with specified initial moments on each atom.

The resulting lowest energy spin configurations have integrated local spin densities of about $\pm 4 \mu_{\mathrm{B}}$ per atom. As might be expected, those atoms with highest coordination have smaller spin densities and the lower-coordinated surface atoms have the highest densities. The icosahedral structures of $\mathrm{Mn}_{13}$ and $\mathrm{Mn}_{19}$ show ferromagnetic coupling within the planes of the 5-membered rings and antiferromagnetic coupling between planes (figure 1). The spin configuration of $\mathrm{Mn}_{15}$ is one in which the corner atoms of the $b c c$ structure are antiferromagnetically coupled with the central and face-centred atoms. For $\mathrm{Mn}_{23}$, the four atoms capping the icosahedral faces are ferromagnetically coupled to the central pentagonal plane.

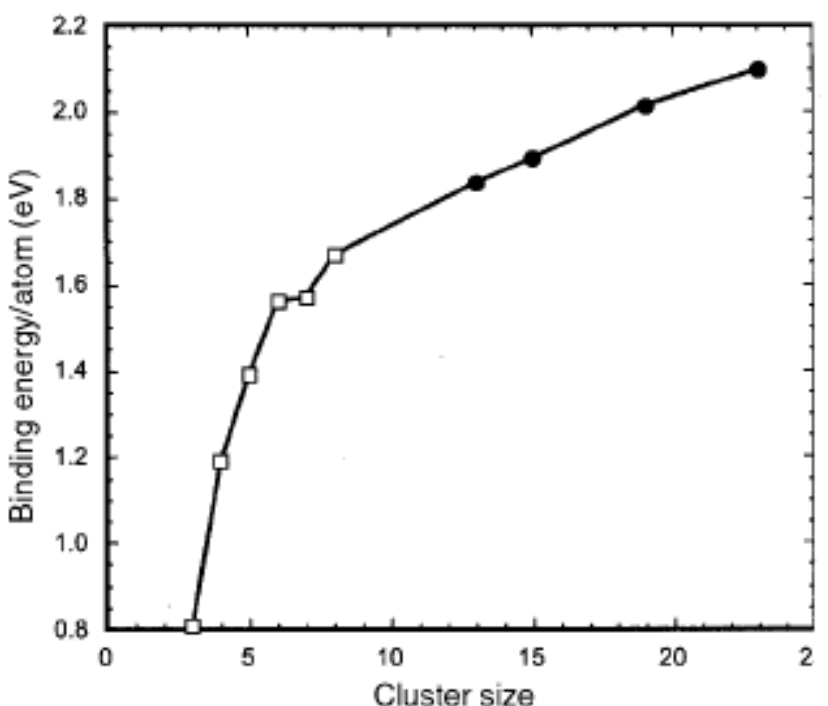

Figure 2. Binding energy per atom as a function of cluster size. The data for $\mathrm{Mn}_{3}-\mathrm{Mn}_{8}$ (empty squares) are taken from Pederson et al (1998). The filled circles are from the present calculation. The bulk cohesive energy is $2.92 \mathrm{eV} /$ atom.

The spin configuration is otherwise similar to that of $\mathrm{Mn}_{19}$. While the most symmetric spin configurations seem to be favoured in general, there is often a small energy difference between this and a less symmetric structure.

\section{Acknowledgements}

The authors would like to thank the staff of the Centre for Computational Materials Science at the Institute for Materials Research for computational assistance. This work was supported in part by a grant-in-aid from the Japan Society for the Promotion of Science. (VK) thankfully acknowledges the kind hospitality at the Institute for Materials Research.

\section{References}

Briere T M, Sluiter M H F, Kumar V and Kawazoe Y 2002 Mater. Trans. $\mathbf{4 3} 424$

Knickelbein M B 2001 Phys. Rev. Lett. 865255

Kresse G and Furthmüller J 1996 Comp. Mater. Sci. 615

Nayak S K, Rao B K and Jena P 1998 J. Phys. Condens. Matter 1010863

Nayak S K, Noojien M and Jena P 1999 J. Phys. Chem. 103 9853

Pederson M R, Reuse F and Khanna S N 1998 Phys. Rev. B58 5632

Perdew J P and Wang Y 1992 Phys. Rev. B45 13244 